POS PROCEEDINGS

\title{
Middle-aged Supernova Remnants with AGILE
}

\author{
A. Giuliani \\ INAF / IASF-Milano, Italy \\ E-mail: giulianieiasf-milano.inaf.it
}

M. Tavani, G. Piano, M. Cardillo, E. Striani, S. Sabatini, A. Argan, E. Costa. E. Del Monte, I. Donnarumma, Y. evengelista, M. Feroci, L. Pacciani, G. Pucella, P. Soffitta, V. Vittorini

INAF / IASF-Roma, Italy

Y. Fukui, A. Kawamura

Department of Astrophysics, Nagoya University, Japan

P. Caraveo, A. Chen, M. Fiorini, S. Mereghetti

INAF / IASF-Milano, Italy

A. Bulgarelli, G. Di Cocco, F. Fuschino, F. Gianotti, C. Labanti, M. Marisaldi, M. Trifoglio

INAF / IASF-Bologna, Italy

F. Verrecchia, C. Pittori, P. Santolamazza, P. Giommi

ASI / ASDC Frascati (Roma), Italy

G. Barbiellini, F. Longo

INFN Trieste, Italy

P. W. Cattaneo, A. Rappoldi

INFN Pavia, Italy

F. D'Ammando, S. Vercellone

INAF / IASF-Bologna, Italy

P. Lipari

INFN Roma I, Italy

A. Pellizzoni, M. Pilia, A. Trois

INAF / Osservatorio Astronomico di Cagliari, Italy 
The GRID instrument onboard the AGILE satellite detected several Supernova Remnants in the energy band E $>100 \mathrm{MeV}$, including the remarkable W28. Here we present an overview of spectral and morphological studies performed for some of them. We show that by combining gamma-ray observations with those at other wavelengths ( $\mathrm{TeV}$ and $\mathrm{CO}(\mathrm{J}=1->0)$ molecular line emission), we can constrain the theoretical models for the gamma ray production in SNRs. We show also that models based on the interaction of hadrons with massive molecular clouds can well reproduce the observations, especially in the case of middle-aged SNRs.

25th Texas Symposium on Relativistic Astrophysics - TEXAS 2010

December 06-10, 2010

Heidelberg, Germany 


\section{SNR W28}

W28 is a mixed morphology SNR with an age of more than 35000 years located at a distance of about $2 \mathrm{kpc}$. A system of massive molecular clouds is associated to the SNR as revealed by the $\mathrm{CO}(\mathrm{J}=0->1)$ observation carried by the NANTEN telescope. Two main peaks in the molecular hydrogen distribution can be seen at R.A., Dec $=270.4,-23.4($ cloud N) and at R.A., Dec $=270.2$, -24.1 (cloud S, see fig. 1)

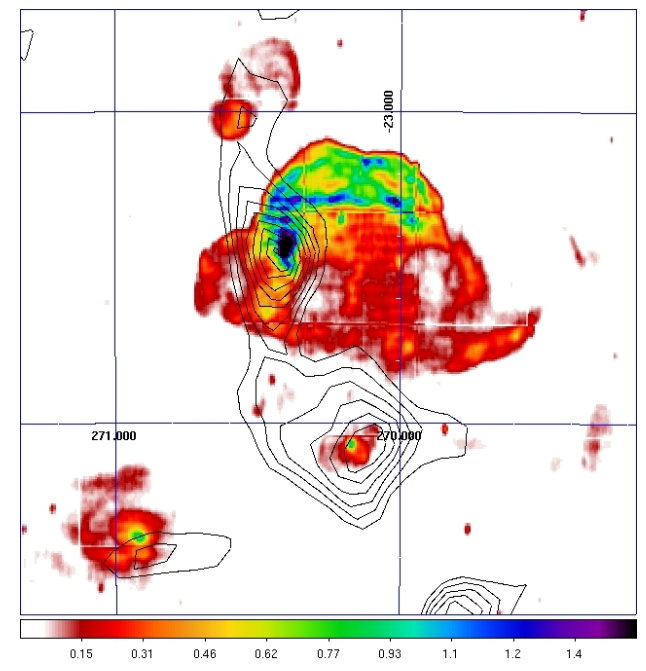

Figure 1: Map of $90 \mathrm{~cm}$ radio emission (VLA) from SNR W28. The black contours show the CO intensity emission integrated over the velocity interval $3-27 \mathrm{~km} / \mathrm{s}$.

The molecular clouds distribution correlates nicely with the gamma rays observations in both the TeV energy band (as observed by HESS, see [1]) and in the E $>400 \mathrm{MeV}$ energy band observed by AGILE. However the ratio between the $\mathrm{TeV}$ and the multi-MeV emission is significantly different for the cloud $\mathrm{N}$ and the cloud S. In figure 3 the gamma-ray spectra for the two clouds are shown.

The interpretative scenario proposed in [2] assumes that the $\mathrm{N}$ cloud is closer to the CR acceleration site than the $\mathrm{S}$ cloud. If protons diffuse in the ISM with a diffusion coefficient given by $\mathrm{D}=\mathrm{D}_{0} \mathrm{E}^{0.5}$ the resulting proton energy spectrum is suppressed below a threshold energy $\mathrm{E}(\mathrm{t})$ $R^{4} t^{-2}$ where $\mathrm{R}$ is the distance from the acceleration site and $\mathrm{t}$ the age of the SNR. Figure 3 shows the gamma-rays spectra produced by protons (through neutral pion decay) interacting with the cloud $\mathrm{N}$ and $\mathrm{S}$ assuming respectively $\mathrm{R}=9$ and $4 \mathrm{pc}$. This scenario can explain also the morphology of the gamma-rays emission seen at different energies ranges (see figure 4). Assuming that $\mathrm{CR}$ are accelerated in a circular region (indicated by the blue circle) we evaluated the tridimensional distribution of CR around the SNR as a function of the particle energy and SNR age. Black countours are the molecular cloud distribution observed by the NANTEN telescope.

The sky maps in the upper row of figure 4 refer to the gamma ray emission for energies $>400$ $\mathrm{MeV},>3 \mathrm{GeV}$ and $>400 \mathrm{GeV}$ expected for an age of $40000 \mathrm{yrs}$. We assumed that gamma ray emission is produced by pp collision between accelerated protons and the nuclei of the molecular 

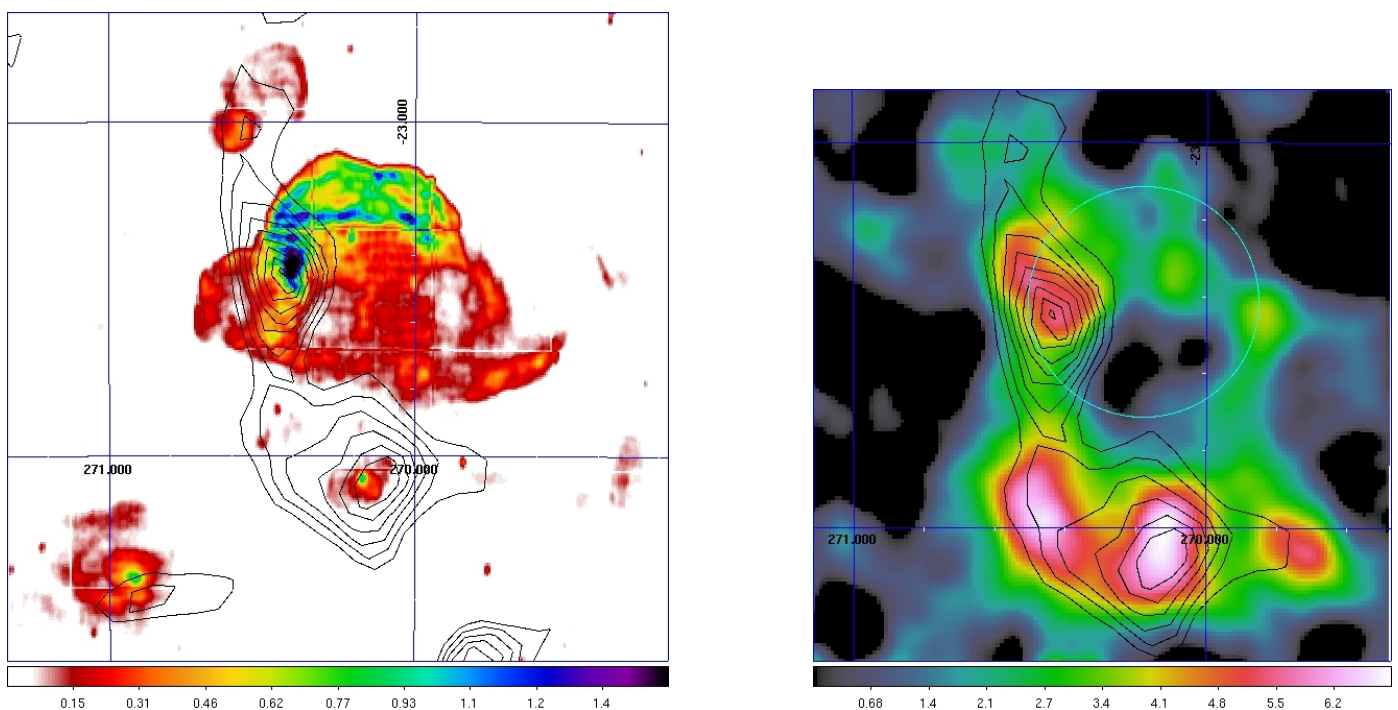

Figure 2: Fig. 2. Left: AGILE counts map for the W28. Right: Significance of the TeV emission detected by the HESS telescope [1]. The blue circles indicate the location of the supernova remnant W28. As in figure 1 the black contours show the $\mathrm{CO}$ intensity emission.

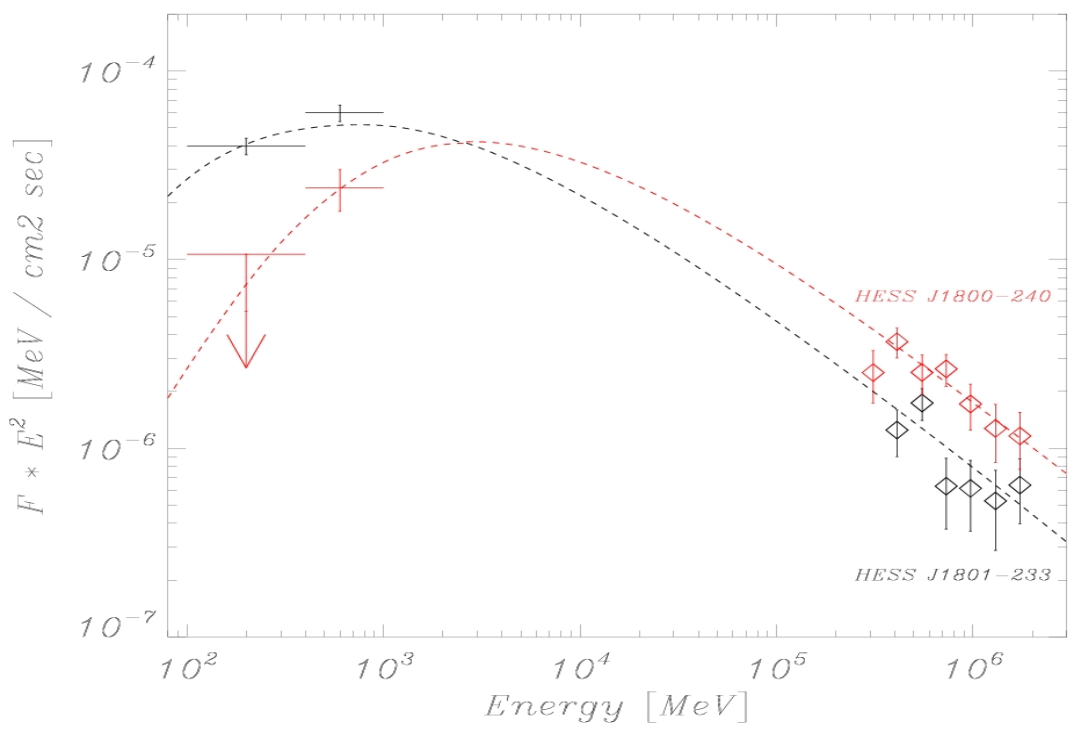

Figure 3: Combined AGILE and HESS gamma-ray photon spectra for cloud N (black) and cloud S (red). The curves represent the gamma-ray spectra estimated (accordling to the model presented in the text) for the two clouds. 


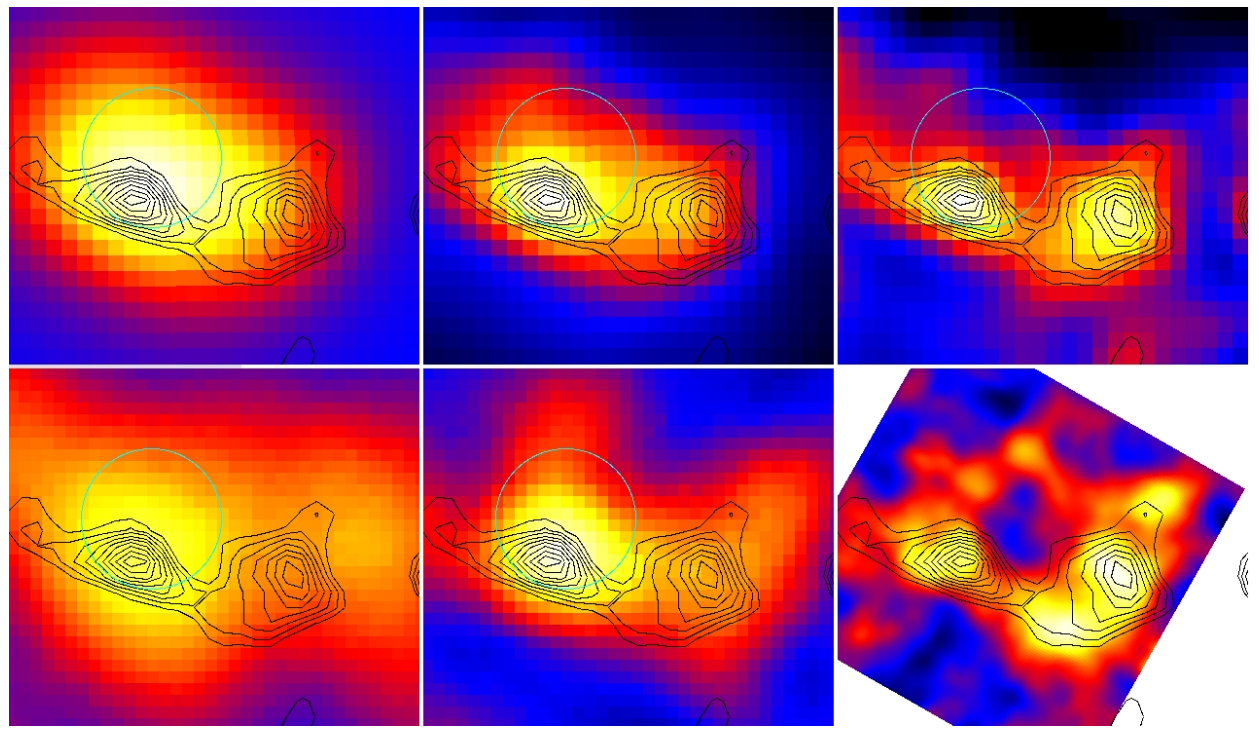

Figure 4: Upper row: Gamma ray emission for energies $>400 \mathrm{MeV},>3 \mathrm{GeV}$ and $>400 \mathrm{GeV}$ expected for W28 after 40000 yrs since the SN explosion. Lower Row: Maps produced by the AGILE, Fermi and HESS in the same energy bands shown in the upper row

hydrogen. In the lower row, the maps produced by the AGILE, Fermi and HESS observations of SNR W28 are shown.

\section{SNR W44}

AGILE detects SNR W44 with a significance of 15.8 sigma, as an extendeted source with a morphology well correlated with the radio shell seen at $74 \mathrm{Mhz}$ (see figure 5). The mesured flux above $400 \mathrm{MeV}$ is $16.0 \pm 1.210^{-8} \mathrm{ph} \mathrm{cm}^{-2} \mathrm{~s}^{-1}$
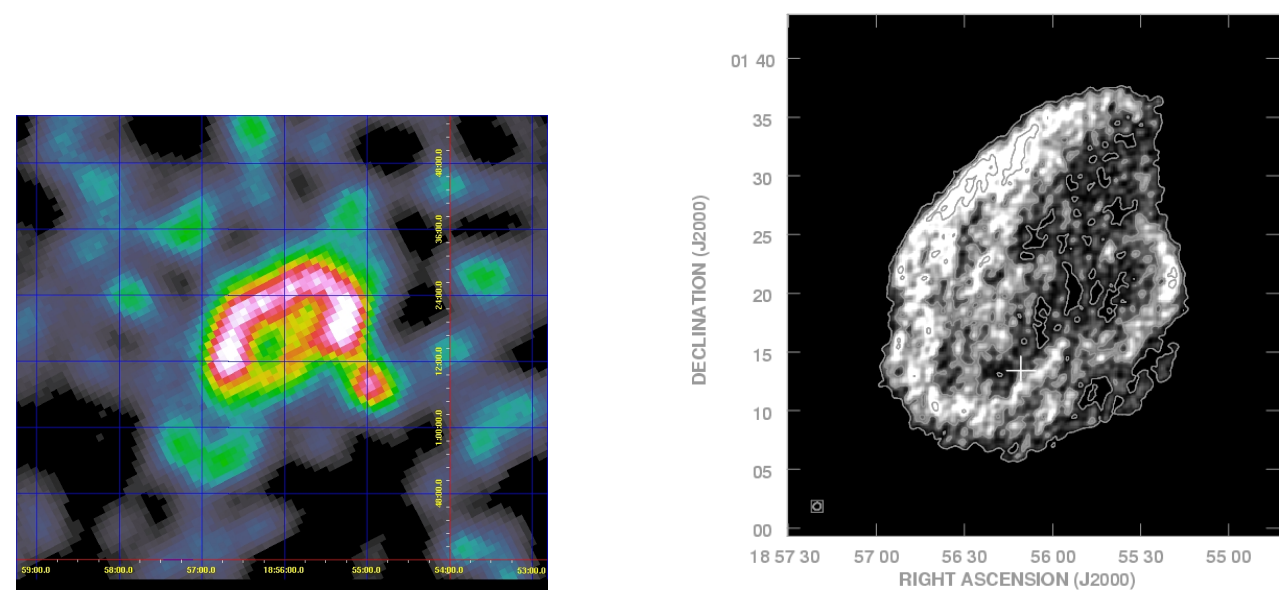

Figure 5: Left: SNR W44 as seen by AGILE for energies greater than $400 \mathrm{MeV}$. Right : VLA map of W44 at $74 \mathrm{Mhz}$ [3] 


\section{SNR W51C}

AGILE detects SNR W51C with a significance of 8.3 sigma and a flux above $400 \mathrm{MeV}$ of $8.9 \pm 1.310^{-8} \mathrm{ph} \mathrm{cm}^{-2} \mathrm{~s}^{-1}$. The shape similar to the radio shell seen at $330 \mathrm{Mhz}$ (see figure 6).
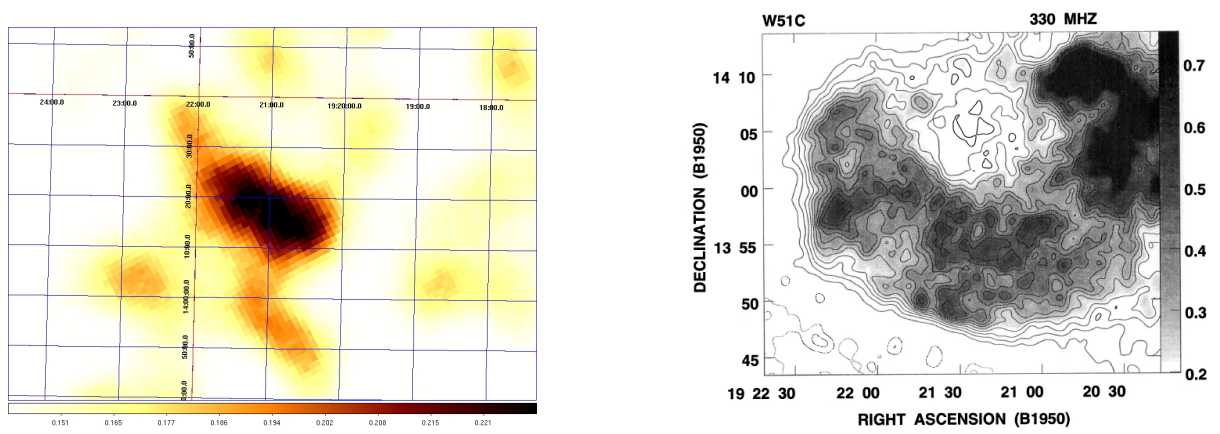

Figure 6: Left : SNR W51C as seen by AGILE for energies greater than $400 \mathrm{MeV}$ Right : VLA map of W51C at $330 \mathrm{Mhz}[4]$

\section{SNR IC 443}

IC 443 is a SNR lying at a distance of about $1.5 \mathrm{kpc}$ in the Galactic anticenter direction. In radio, optical and X rays a shell structure is clearly visible, where the interaction of the SNR and the ISM creates a shock. A system of molecular clouds is also associated to the SNR, and an evidence of interaction is given by the observations of an high value of the ratio $\mathrm{CO}(\mathrm{J}=2-1) /(\mathrm{J}=1-0)$ [5].

EGRET observed a source in the direction of IC 443 in the $100 \mathrm{MeV}-10 \mathrm{GeV}$ energy range, suggesting the possibility that the enhanced cosmic ray flux produced by the shock interacts with the clouds and produces gamma-ray through pion decay and electron bremmstrahlung. A TeV

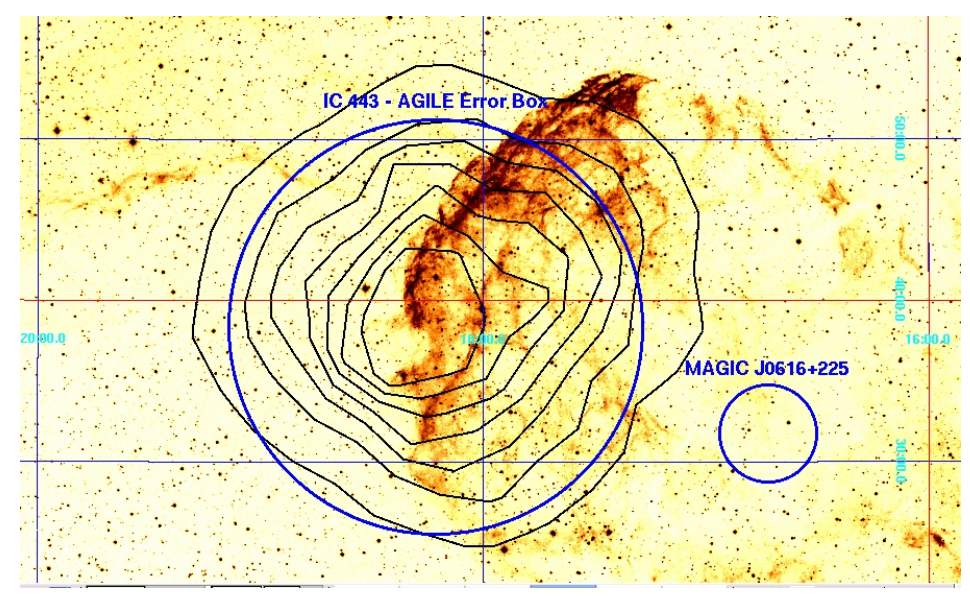

Figure 7: An optical image of IC 443. The contours are the AGILE confidence levels. The $95 \%$ c.l. error boxes of AGILE and MAGIC are also shown. 


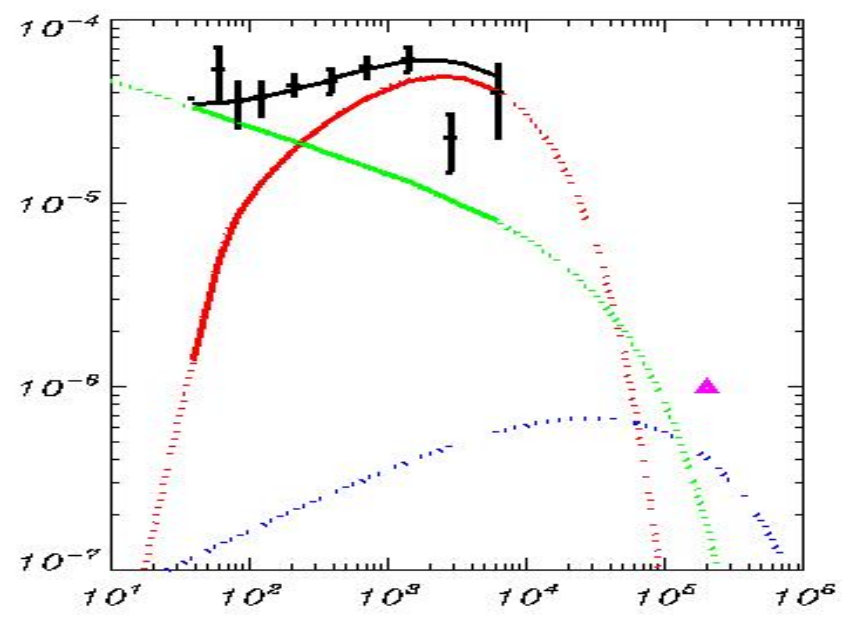

Figure 8: The spectrum of IC 443 can be modeled by a pion-decay component (red) plus an electron bremsstrahlung component (green).

source has been detected by MAGIC [6]. Due to the good angular resolution of the MAGIC telescope it was possible to locate the source in a small error box coincident with the direction of the most massive cloud. AGILE observed a gamma-ray source in this direction in the same energy range of EGRET, obtaining an error box which is not compatible with the MAGIC error box [7]. The different position of the source in the $\mathrm{TeV}$ and gamma energy ranges implies a difference in the the CR spectrum in the two emitting places or in the targets distributions. A possible interpretation can be given assuming a different distance of the emitting clouds which can lead to a different spectrum of the accelerated protons seen by the near/far clouds [8].

\section{References}

[1] F. Aharonian et al., $A \& A$ 481, 2008

[2] A. Giuliani et al., $A \& A$ 516, 2010

[3] G. Castelletti et al., $A \& A$ 471, 2007

[4] R. Subrahmanyan \& W. Goss, MNRAS, 275, 1995

[5] M. Seta et al., ApJ, 505, 1998

[6] M. Tavani et al., ApJ, 710, 2010

[7] J. Albert et al., ApJ, 664, 2007

[8] F. Aharonian \& Atoyan, A\&A, 309, 1996 